\title{
PULSE FORMING IN AN AM MODE-LOCKING HYBRID TEA-CO, LASER
}

\author{
R.J.M. BONNIE and F.A. VAN GOOR \\ Department of Applied Physics, Twente University of Technology, Enschede, The Netherlands
}

Received 25 November 1985; revised manuscript received 17 January 1986

\begin{abstract}
We performed an experimental study on the pulse forming mechanism in an AM mode-locked hybrid TEA-CO $\mathrm{CO}_{2}$ laser by means of "injection mode-locking". The results show that bandwidth limited pulses will only be reached within a small critical - range of parameters. Furthermore, under some stringent conditions, the pulse-widths obtained become comparable to the non-hybrid case. We demonstrate also that the relation between the pulse-width and the modulation-depth is not different from a non-hybrid $\mathrm{AM}$ mode-locked TEA- $\mathrm{CO}_{2}$ laser.
\end{abstract}

\section{Introduction}

A widely accepted method for generating short pulses in the nanosecond region in (pulsed) $\mathrm{CO}_{2}$ lasers is active mode-locking by means of an intra cavity acousto-optic modulator. It can be shown that in a typical AM mode-lock experiment, pulse widths in the order of $1 \mathrm{~ns}$ are feasible.

Because the laser starts to oscillate from the spontaneous emission induced noise, a large number of rondtrips is needed (especially at low values of the modulation depth) to produce stable and reproducible pulses. This requirement is not always fulfilled when dealing with TEA-CO ${ }_{2}$ lasers. To overcome this problem, several stabilization techniques have been developed. The easiest is the inclusion of a low pressure $\mathrm{cw}$-section in the cavity [1]. This mode-locking of a hybrid TEA-CO $\mathrm{CO}_{2}$ laser offers the facility to produce pulses with variable durations between 1 and 10 ns. A disadvantage of this technique however is the additional broadening of the pulses compared to the non-hybrid case.

Injection of monochromatic radiation from a cw$\mathrm{CO}_{2}$ laser in an AM mode-locked TEA-CO 2 laser results in very stable pulses without the disadvantage of broadening [2]. On the other hand, this technique appears to depend critical on a proper frequency matching between both lasers.

Three observations have strongly encouraged us to study the pulse forming in a hybrid AM mode-locked TEA-CO ${ }_{2}$ laser:

(i) The additional broadening of the pulses from the mode-locked laser, when dealing with an intracavity $\mathrm{cw}-\mathrm{CO}_{2}$ stabilization cell.

(ii) Mode-locking of a hybrid laser results in a pulsewidth varying with the modulation depth $\delta_{\mathrm{m}}$ as $\delta_{\mathrm{m}}^{-0.55}$, in contrast to the $\delta_{\mathrm{m}}^{-0.25}$ dependence in the non-hybrid case [1].

(iii) Pulse-durations in rich mixtures - i.e. mixtures containing little helium - can be considerably longer than in lean mixtures (containing much helium). Unless the observed pulses are not bandwidth limited, this is in contradiction with the generally accepted equation for the bandwidth of a typical $\mathrm{CO}_{2}$ laser [3]; according to this, bandwidth increases with decreasing helium percentage. Hence, the pulse duration should increase with increasing helium percentage.

\section{Description of the experiment}

Injection of the radiation from the AM modelocked hybrid TEA-CO $\mathrm{CO}_{2}$ laser in a high pressure TE$\mathrm{CO}_{2}$ regenerative amplifier at various delay-times offers the opportunity to study the time evolution of the pulse running in this oscillator. Using this tech. nique it is possible to perform measurements even in those regions where the radiation level is too low to 


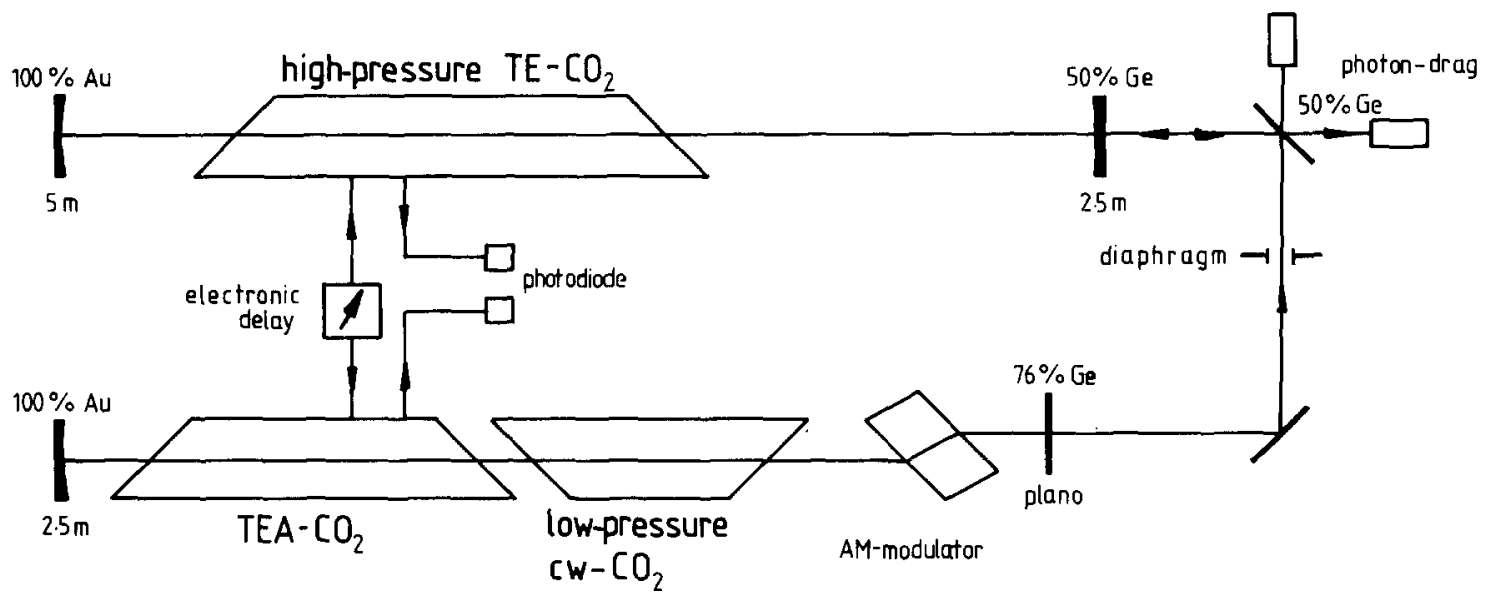

Fig. 1. Experimental configuration.

detect with any kind of available detector.

The experimental configuration is shown in fig. 1 .

Because we already discussed this setup in detail [4], we shall give a brief description only.

The injection source is formed by a single-discharge TEA-CO $\mathrm{CO}_{2}$ section with an active volume of $20 \times 1 \times 1$ $\mathrm{cm}^{3}$, a $55 \mathrm{~cm}$ long low pressure section filled with 20 Torr of a $\mathrm{CO}_{2}: \mathrm{N}_{2}: \mathrm{He}=1: 2: 5$ mixture and a $40 \mathrm{MHz}$ acousto-optic AM-modulator. The amplifier is a double-discharge, UV-preionized TE- $\mathrm{CO}_{2}$ laser with an active volume of $30 \times 0.8 \times 0.8 \mathrm{~cm}^{3}$, operating on 5 atmospheres of a $\mathrm{CO}_{2}: \mathrm{N}_{2}: \mathrm{He}=1: 1: 10$ mixture. This high pressure results in an effective bandwidth of roughly $25 \mathrm{GHz}$, sufficiently large to minimize the effect of broadening or distortion of the injected pulses by the (dispersive) amplifying medium. The radiation is injected in the amplifier through its outcoupling mirror. The output of both the injector and the amplifier is monitored via two photon-drag detectors on a Tektronix $7912 \mathrm{AD}$ transient digitizer.

The delay between both lasers can be varied electronically. To circumvent the effect of jitter in the triggering of the lasers, we also measured the delay by observing the light-signals coming from the last sparkgaps of the Marx-generators of the lasers.

We measured under several experimental conditions the pulse-duration as a function of the delay time; the results of a typical measurement are shown in fig. 2. The intensity profile of the envelope of the output pulse from the oscillator, as detected by

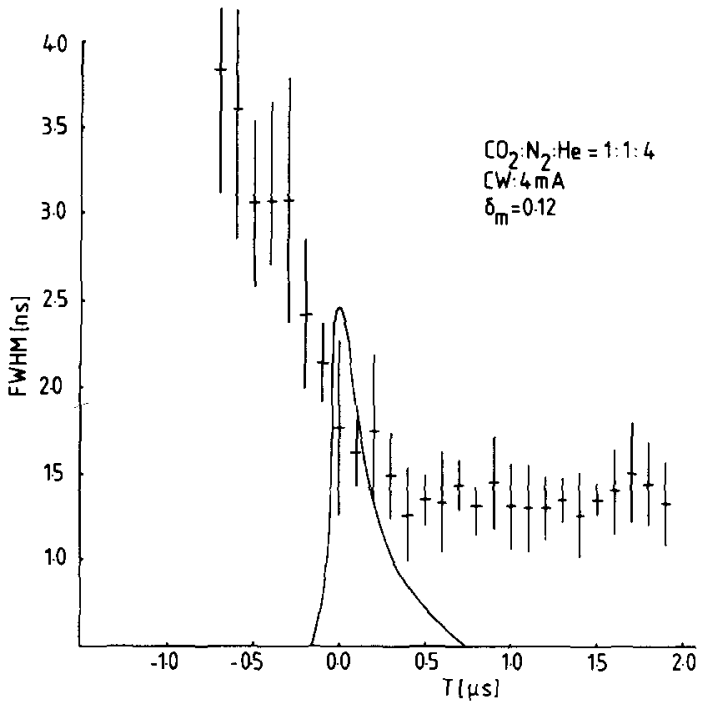

Fig. 2. Duration of the mode-locked pulses as a function of time; the intensity profile of the envelope of the pulse-train as detected by means of a photon-drag detector is also shown; $t=0$ is chosen to coincide with the maximum of the intensity profile.

means of a photon-drag detector, is also shown. We have chosen the time scale in such a way that the maximum of this intensity profile coincides with $T=0$. 


\section{Experimental results and discussion}

\subsection{Pulse-width as a function of the helium concentration}

When the pulse-width is measured directly by means of e.g. a photon-drag detector, one has to be careful in interpretating the results. As is demonstrated clearly in fig. 2 , the time necessary for the pulseforming to reach steady state may be considerably longer than the build-up time for the radiation field. For this reason, the measured width on its turn can be larger than expected from steady-state theory.

Keeping the ratio $\mathrm{CO}_{2}: \mathrm{N}_{2}=1: 1$, we measured as a function of the helium percentage for two values of the modulation depth parameter $\left(\delta_{m}\right)$ the pulse-width directly $\left(\tau_{m}\right)$ - i.e. at the moment of output intensity was at maximum - and also in steady state $\left(\tau_{\mathrm{s}}\right)$. The results are shown in figs. $3 a$ and $3 b$.

Restricting to $\tau_{\mathrm{m}}$, one could conclude that the

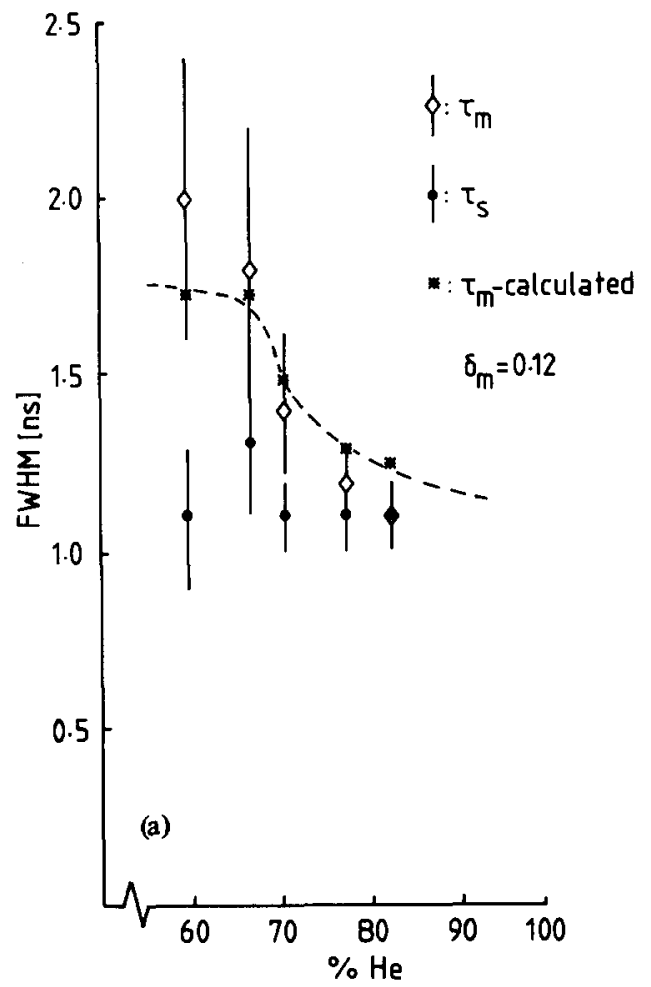

pulse-width indeed decreases with increasing helium percentage; however, the values of $\tau_{s}$ indicate that the pulse-width is independent from the helium concentration (there may be a small dependence, but this is within the experimental error).

These observations can be explained as follows. Decreasing the amount of helium in the laser gas mixtures increases the gain (keeping the other experimental parameters unchanged). So less roundtrips are needed before the laser passes threshold and the gain switches. Thus there is also less time available for the pulse forming process. This time may be insufficient to reach steady state and the measured pulse durations may be longer than expected from steady-state theory. To check this interpretation, we measured the delay between the pump-pulse of the laser and the optical output. The results are shown in fig. 4 . As expected, the delay increases with increasing helium percentage.

The transient behaviour of the pulse width from

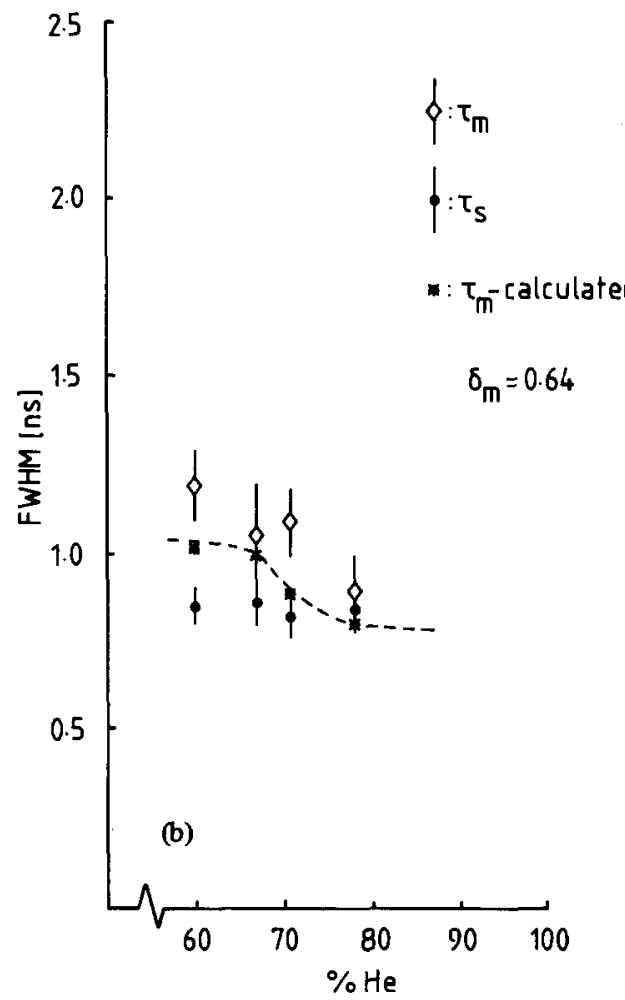

Fig. 3. Pulse width measured in the maximum of the intensity profile $\left(\tau_{m}\right)$ and in steady-state $\left(\tau_{\S}\right)$ as a function of the helium percentage. Calculations for $\tau_{\mathbf{m}}$ according to eq. (1) are also shown. 


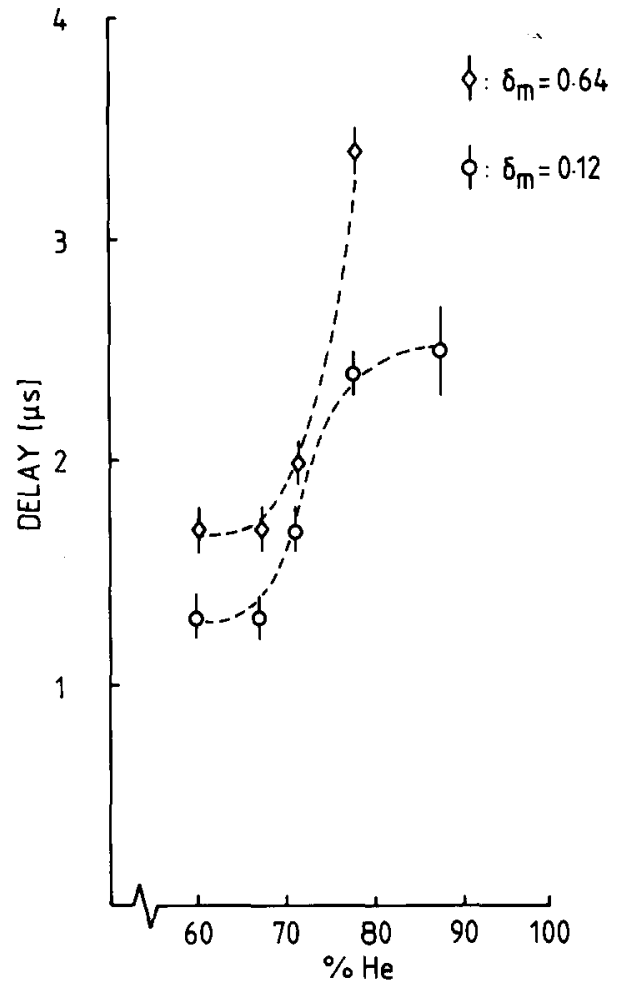

Fig. 4. Delay between the electrical pump pulse and the most intense pulse in the train for different helium percentages. an AM mode-locked laser can - in analytical form be described by [5]

$\tau=\tau_{\mathrm{s}} /\left[\tanh \left(t / t_{0}\right)\right]^{1 / 2}$,

where $\tau_{\mathrm{s}}$ is the steady state pulse width,

$t_{0}=(\Delta f) / 8 \sqrt{2} g^{1 / 2} \delta_{\mathrm{m}}^{1 / 2} f_{\mathrm{m}}^{2}$,

$\delta_{\mathrm{m}}$ is the modulation depth, $g$ is the steady state gain, $f_{\mathrm{m}}$ is the AM modulator drive frequency, and $\Delta f$ is the bandwidth of the medium.

Using both these delays between the pump-pulse and the appearance of the most intense pulse in the output train and the steady-state pulse duration at various helium percentages, we may calculate according to eq. (1) the width of the most intense pulse as a function of the helium concentration. The calculated curves are also shown in the figs. $3 a$ and $3 b$.

\subsection{Pulse width as a function of the modulation depth}

We measured at three different places in the pulse train the pulse duration as a function of the modulation depth $\left(\delta_{\mathrm{m}}\right): \tau_{\mathrm{s}}$ pulse width in steady state, $\tau_{\mathrm{m}}$ width of the strongest pulse in the train, and $\tau_{\mathrm{d}}$ width of one of the first directly detectable pulses - i.e. 0.4 $\mu$ sefore the strongest pulse.

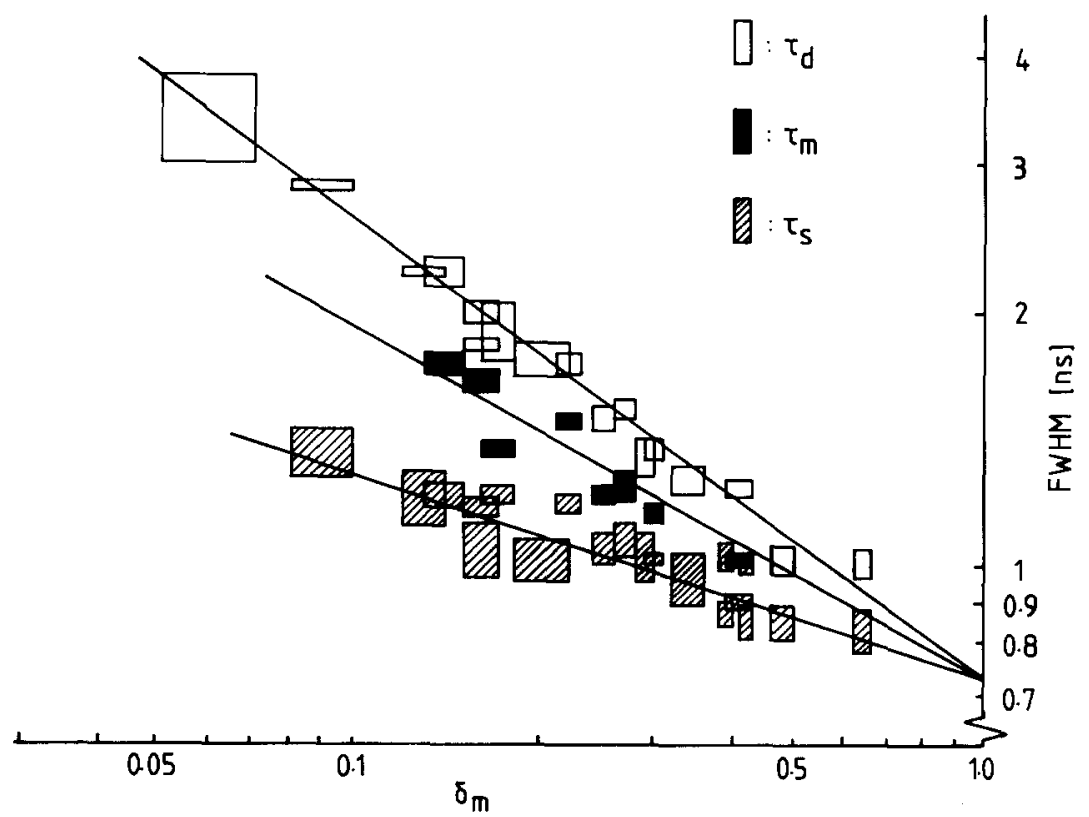

Fig. 5. Variation of pulse duration $\tau$ with the modulation depth $\delta \mathrm{m}$. The full lines are the best straight-line-fits through the data. See text for an explanation of the indices $\mathrm{d}, \mathrm{m}, \mathrm{s}$. 


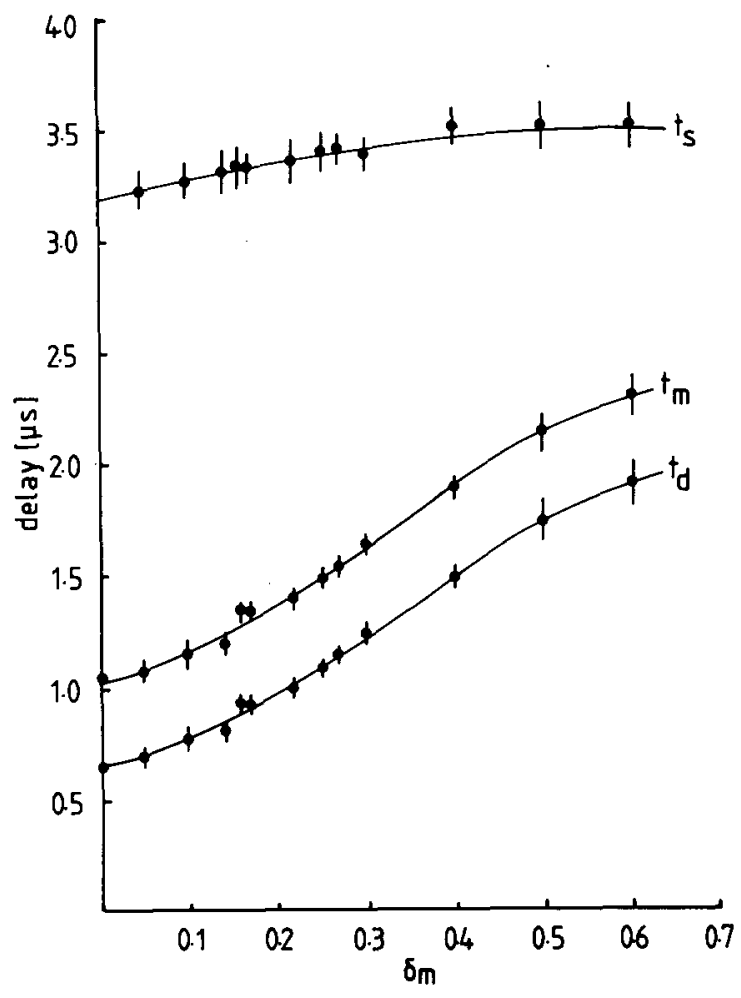

Fig. 6. Delay measurements corresponding to the pulse width measurements of fig. 5 .
Because we are interested in the relation between $\tau$ and $\delta_{\mathrm{m}}$, we plotted in fig. $5 \tau$ versus $\delta_{\mathrm{m}}$ on a double logarithmic scale; also the best straight-line-fit through the data has been drawn. From these results, we obtain the following relation:

$\tau_{i}=\tau_{0} \delta_{\mathrm{m}}^{-\alpha_{i}}$,

where $i$ is $\mathrm{d}, \mathrm{m}, \mathrm{s}$, respectively. $\tau_{0}$ is the observed steady-state pulse-width for $\delta_{\mathrm{m}}=1 ; \tau_{0}=0.74(4) \mathrm{ns}$,

$\alpha_{d}=0.55(3), \quad \alpha_{m}=0.43(3), \quad \alpha_{s}=0.25(1)$;

note that these results are for a $\mathrm{CO}_{2}: \mathrm{N}_{2}: \mathrm{He}=1: 1: 3$ mixture.

It is seen from this figure that the value of the parameter $\alpha$ in formula (2) strongly depends on the position of the pulse in the output-pulse-train. More exactly, the parameter $\alpha_{i}$ is correlated with the delay between the electrical pump-pulse of the laser and the $i$ th pulse in the train. For the three cases as described above, we measured - for the same mixture - also the delay as a function of the modulation depth. The results are shown in fig. 6 . From these delay measurements we constructed fig. 7 according to [5] $\tau=\tau_{0} \tau_{\mathrm{m}}^{-1 / 4} /\left[\tanh \left(t / t_{0}\right)\right]^{1 / 2}$.

Next we wish to fit the data depicted in fig. 7 with

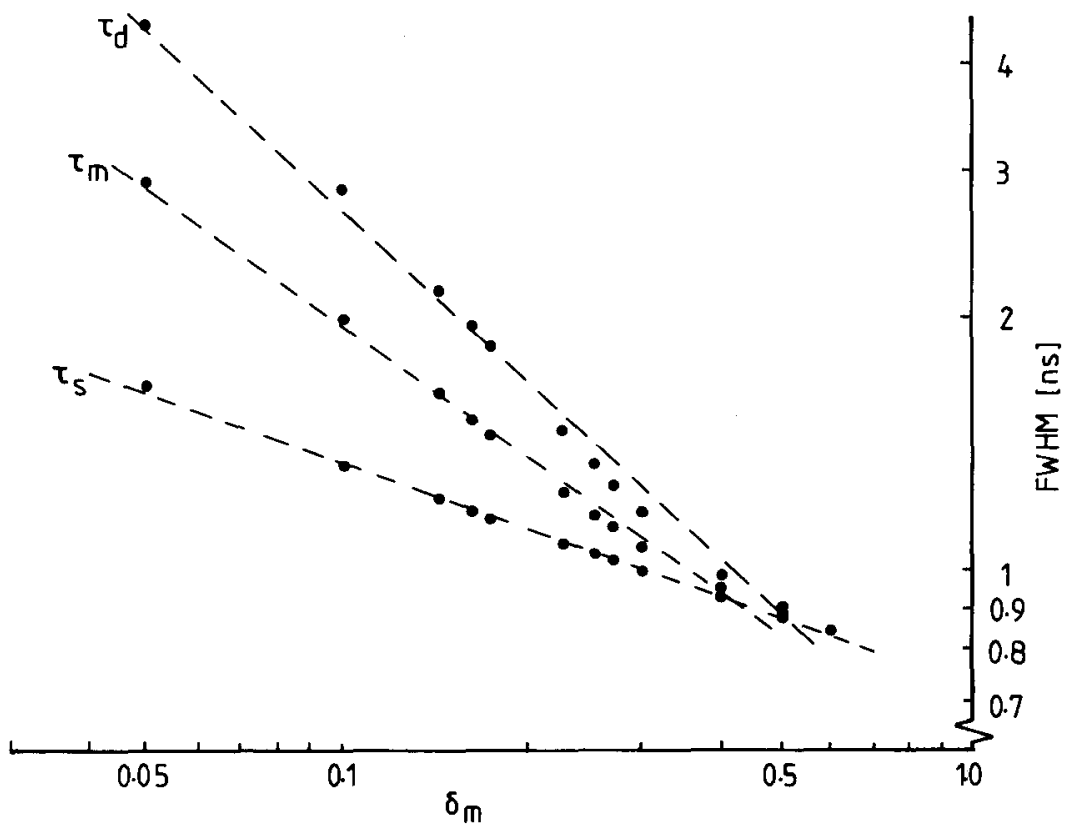

Fig. 7. Calculated variations of the pulse width with the modulation depth according to eq. (4), using the delay measurements of fig. 6 . 
relation (2); from this first order fit, we obtain:

$\alpha_{d}=0.55(2), \quad \alpha_{m}=0.47(2), \alpha_{s}=0.28(2)$.

Comparison of these results to those obtained before (3) shows that the exponential behaviour is quite well described by the transient solution of Siegmann and Kuizenga (eq. (4)).

Hence, we might explain the unexpected $\delta-0.55$ behaviour of ref. [1] as caused by the fact that the pulse forming process in the laser system used has not already reached steady state at the moment of detection of the pulses.

\subsection{Additional broadening due to the stabilization cell}

We performed also some measurements with the low pressure $\mathrm{cw}-\mathrm{CO}_{2}$ section switched off. We determined the steady-state pulse-duration as a function of the modulation depth. Comparising these results to the ones obtained with the stabilization cell active, we measured only a very small broadening

$\left(\tau_{\mathrm{s}}\right)_{\mathrm{cw}-\mathrm{on}}=1.06(2) *\left(\tau_{\mathrm{s}}\right)_{\mathrm{cw} \text {-off }} \cdot$

This small additional broadening might be explained as a rest-effect due to the cw-section; i.e. the low pressure gain is not completely depleted in the steady state. The resulting effective bandwidth of both the TEA laser and the low pressure cell will then be smaller, yielding an enlarged pulse width.

\section{Conclusions}

Summarizing the present results, we come to the following conclusions:
- Pulses produced during (or before) the gain switch in an AM mode-locked hybrid TEA-CO ${ }_{2}$ laser may not be bandwidth limited, especially when dealing with a high gain system.

- The broadening of the pulses from an AM modelocked hybrid TEA-CO ${ }_{2}$ laser is neglectable compared to the pulses from a non-hybrid system under the assumption that enough time is available for the pulse forming to approach steady state. This can be established by using low-gain gas mixtures (high percentage helium), low pumping rates or high cavity loss factors (low outcoupling reflection).

- The pulse-width varying with the modulation parameter $\delta_{\mathrm{m}}$ as $\delta_{\mathrm{m}}^{-0.55}$ as reported in ref. [1] can be explained by the assumption that the pulse-forming process has not reached steady state. Looking at different stages of the pulse-forming process, we obtain different values for the exponential dependence on the modulation parameter $\delta_{\mathrm{m}}$.

\section{Acknowledgements}

This investigation was made possible by the financial support of the Dutch "Stichting voor Fundamenteel Onderzoek der Materie".

\section{References}

[1] P. Bernard and P.A. Bélanger, Optics Lett. 4 (1979) 196.

[2] F.A. van Goor, Optics Comm. 45 (1983) 404.

[3] R.L. Abrams, Appl. Phys. Lett. 25 (1974) 609.

[4] F.A. van Goor, R.J.M. Bonnie and W J. Witteman, IEEE J. Quant. Electron. QE-21 (1983) 1772.

[5] A.E. Siegman and D.J. Kuizenga, Opto-Electron 5 (1984) 43. 